\title{
Finite Element Modeling and Validation of Metal Deposition in Wire Arc Additive Manufacturing
}

\author{
Akram Chergui ${ }^{(凶)}$, Nicolas Beraud, Frédéric Vignat, and François Villeneuve \\ Univ. Grenoble Alpes, Grenoble INP, G-SCOP, 46 Avenue Felix Viallet, 38031 Grenoble, France \\ Mohammed-akram. chergui@grenoble-inp.fr
}

\begin{abstract}
Wire arc additive manufacturing allows the production of metallic parts by depositing beads of weld metal using arc-welding technologies. This low-cost additive manufacturing technology has the ability to manufacture large-scale parts at a high deposition rate. However, the quality of the obtained parts is greatly affected by the various thermal phenomena present during the manufacturing process. Numerical simulation remains an effective tool for studying such phenomena. In this work, a new finite element technique is proposed in order to model metal deposition in WAAM process. This technique allows to gradually construct the mesh representing the deposited regions along the deposition path. The heat source model proposed by Goldak is adapted and combined with the proposed metal deposition technique taking into account the energy distribution between filler material and the molten pool. The effectiveness of the proposed method is validated by series of experiments, of which an example is detailed in this paper.
\end{abstract}

Keywords: Wire-arc-additive-manufacturing $\cdot$ Metal deposition $\cdot$ Finite element . Thermal behavior

\section{Introduction}

Wire Arc Additive Manufacturing (WAAM) is one of the most promising additive manufacturing (AM) processes. It combines an electrical arc as a heat source and a wire as feedstock, and parts are obtained by depositing weld beads, layer-by-layer, using arc welding technologies. This technology is inexpensive, offers a large workspace, and allows high deposition rates. However, the quality of parts manufactured using WAAM is greatly affected by the various thermal phenomena present during the manufacturing process. Béraud et al. [1] showed that process simulation remains an efficient tool for building a more thorough understanding of the different thermal phenomena involved in metal additive manufacturing. This method allows not only to reduce experimentation, but also to better optimize the process at a lower cost. Most recent work in additive manufacturing literature addressed process simulation using finite element method. Xiong et al. $[4,5]$ studied the thermal behavior of cylindrical parts made by WAAM through a finite element thermal simulation of the process. Montevecchi et al. [6, 7] developed a finite element model based on a mesh coarsening technique in order to reduce the computational cost of the process simulation. In the same perspective, Ding et al. [10]

(C) The Author(s) 2021

L. Roucoules et al. (Eds.): JCM 2020, LNME, pp. 61-66, 2021.

https://doi.org/10.1007/978-3-030-70566-4_11 
proposed a finite element approach based on two models (transient and stationary) in order to investigate the thermomechanical behavior of parts manufactured in WAAM. Michaleris [9] reviewed the existing techniques for metal deposition modeling, and proposed a new hybrid algorithm in order to reduce the computational time.

From the perspective of a simulation, WAAM process is very similar to multi-pass welding process [6]. However, the physics of welding process put forward some complex physical phenomena, involving thermodynamics, heat and mass transfer, electricity and magnetism [11]. Modeling such phenomena at a part scale level can be costly due to the unreasonable computational time requirements. For this reason, most studies in literature recommend to model the heat input using a heat source model, taking into consideration the energy contribution of the different physical phenomena occurring in the molten pool. In this article, the double-ellipsoid heat source model proposed by Goldak [3] is adapted and combined with a new finite-element metal deposition technique in order to model material and heat input in WAAM process simulation. The proposed method allows not only to take into account the energy distribution between filler material and the molten pool, but also to consider the changing in the boundary conditions during the deposition process. The overall model is validated with an experimental test case, and the results are presented.

\section{Proposed FE Metal Deposition Technique}

\subsection{Metal Deposition Modelling}

There are two finite element techniques for modeling material deposition in additive manufacturing process simulation: inactive element method and quiet element method, as reviewed in literature by Michaleris [9]. In the inactive element method, the elements representing the deposited regions are initially inactive, and activated gradually according to the deposition path. In the quiet element method, all elements are present from the start of the analysis, but low values are assigned to their material properties (conductivity and specific heat), so they do not affect the analysis. These material properties are then switched to the real values according to the deposition path. Both inactive and quiet methods can be used to model metal deposition in WAAM process, and each presents some advantages over the other. But also some disadvantages such as introducing errors into the finite element analysis, and taking a longer time to run. Furthermore, the interface between inactive (or quiet) and active elements is continuously evolving during the deposition phase, making it difficult to compute this internal interface and consider its surface convection and radiation. For this reason, convection and radiation on this interface are neglected on both inactive and quiet element techniques. Leading to additional errors in the finite element analysis [9] (Figs. 1 and 2).

In the present model, metal deposition is taken into account using a new element deposition technique. In this technique, each deposited droplet is modeled by a set of elements, representing together a numerical droplet. Every numerical droplet is created at its corresponding time-step in the deposition phase simulation. The new created elements are then added to the elements already created in the previous time-steps. Thus, the mesh representing the deposited regions is constructed gradually along the deposition path. 


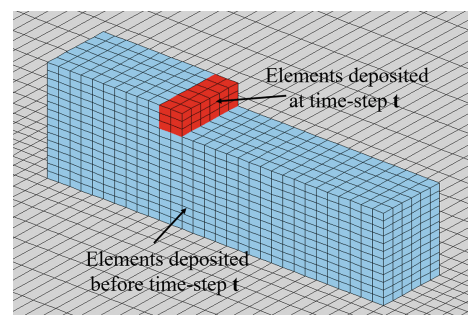

Fig. 1. Element deposition technique

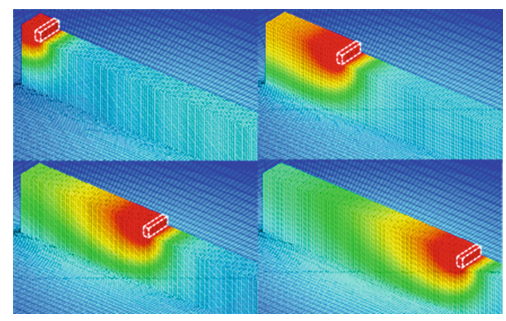

Fig. 2. Mesh evolution using the element deposition technique.

\subsection{Heat Input Modelling}

Goldak et al. [3] proposed a double-ellipsoid heat source model in order to model the heat input in welding process. The model is expressed as follows:

$$
q_{f, r}(x, y, z)=\frac{\left(6 \sqrt{3} f_{f, r} Q\right)}{\left(\pi a b c_{f, r} \sqrt{\pi}\right)} \exp \left(-3 \frac{x^{2}}{a^{2}}-3 \frac{y^{2}}{b^{2}}-3 \frac{z^{2}}{c_{f, r}^{2}}\right)
$$

Where $q_{f}$ and $q_{r}$ are the heat flux densities of the front and rear ellipsoids respectively. $a, b c_{f}$ and $c_{r}$ are the semi-axes of the two ellipsoids. $Q$ is the arc energy input, and $Q=\eta U I . I, U$ and $\eta$ are the welding current, voltage and efficiency. $f_{f}$ and $f_{r}$ are the distribution factors of the heat flux in the front and rear ellipsoids, and $f_{f}+f_{r}=2$.

In WAAM, the arc power is not fully delivered to the part directly. According to previous works, about $50 \%$ of the total arc power is used to melt the feed wire. This energy is subsequently transmitted to the part through the enthalpy of the deposited droplet [6] (Figs. 3 and 4)

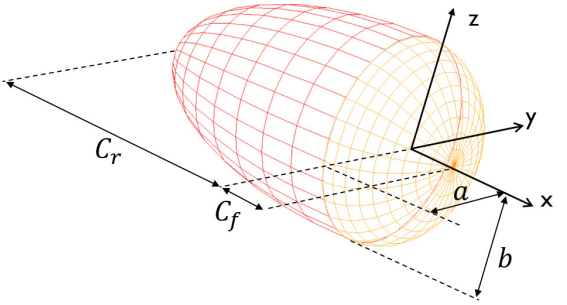

Fig. 3. Goldak heat source

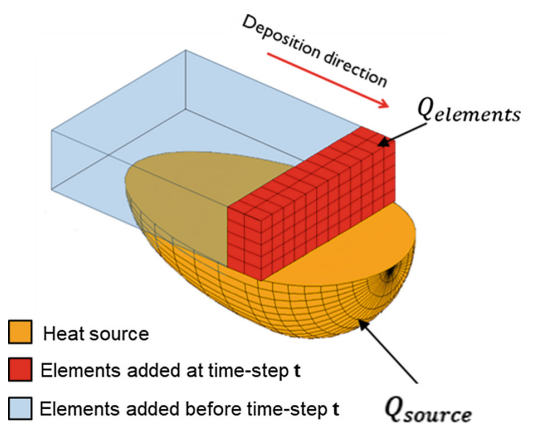

Fig. 4. Adapted Goldak heat source

In the present model, the Goldak heat source is adapted and combined with the proposed element deposition technique to model the heat input in WAAM process, taking into consideration this energy distribution between the wire and the substrate. In fact, the direct energy transfer from the arc to the substrate is considered using the inferior half of the double-ellipsoid Goldak model. The remaining 50\% of the total energy is 
delivered by means of the deposited elements. These latter are charged with an amount of energy equivalent to the energy delivered by the adapted heat source, and expressed as follows:

$$
Q_{\text {source }}=Q_{\text {elements }}=\frac{\eta U I}{2}
$$

\subsection{Model Update}

As discussed earlier, the internal interface between inactive (or quiet) and active elements is difficult to compute, because it changes continuously during the deposition phase. Therefore, surface convection and radiation are often neglected on this interface using inactive and quiet element methods, thus introducing errors to the finite element analysis. It is easier to overcome such issue using the proposed element deposition technique, as the internal active/inactive interface becomes an external free surface. In the proposed simulation, a model-update procedure is developed in order to update the boundary conditions and the material properties after each elements deposition step. This procedure allows to compute the external surface of the total mesh, to apply surface convection and radiation adequately, and to assign the relevant material properties to the new-deposited elements. The evolution of material properties such as conductivity, specific heat and density as a function of temperature is also taken into consideration, and the latent heat is considered as a triangular variation of the specific heat between solidus and liquids temperatures.

\section{Validation}

In order to check the effectiveness of the proposed technique, an experimental validation was carried out by comparing simulated and experimental thermal curves of a test case. The test case consists of fabricating two thin-walled parts of eight layers according to two different strategies: raster and zigzag. Temperatures as function of time were measured at six different points (P1, P2 and P6) on the substrate plate using six k-type thermocouples.

The test case was carried out using a WAAM cell composed of a Yaskawa DX200 6-axis robot, a Fronuis CMT welding technology, and a 2-axis positioner. Two different materials have been considered in this experiment. The substrate plate material was a 5083 welding aluminum alloy, and the thin-walls structures were manufactured using a 5356 aluminum alloy filler wire with a diameter of $1.2 \mathrm{~mm}$. The beads were deposited under a $100 \%$ argon gas protection with a flow rate of $13 \mathrm{~L} / \mathrm{min}$ (Fig. 5).

\subsection{Results}

Figure 6 presents the experimental temperature curves as a function of time at points P1 $\mathrm{P} 2$ and P3, compared to the results obtained with the proposed simulation. Comparison for both Raster and Zigzag strategies are shown in Fig. 6(a) and Fig. 6b, respectively. 

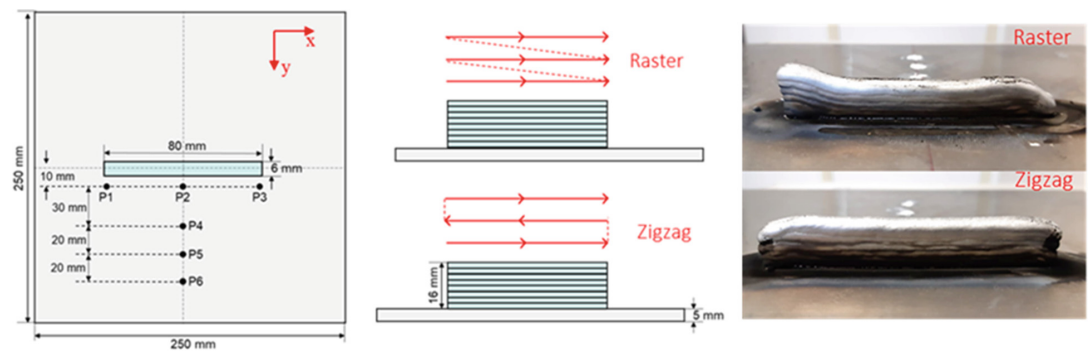

Fig. 5. Experimental design
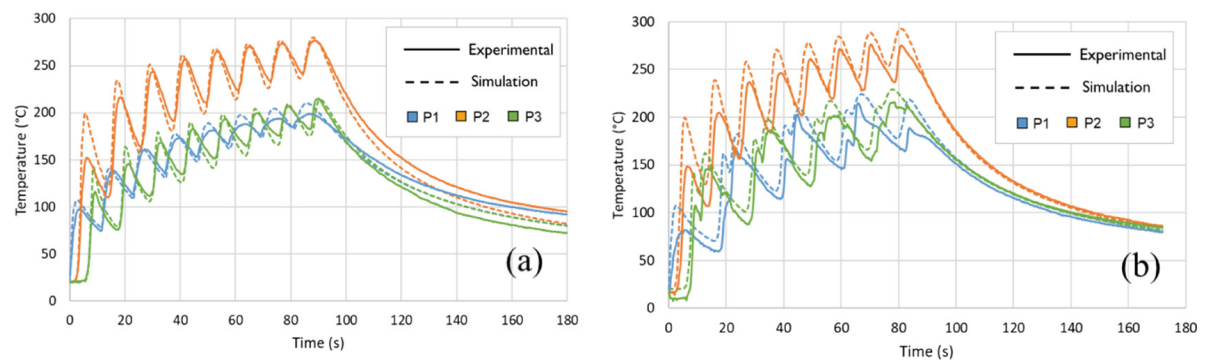

Fig. 6. Temperature curves comparison between the experimental measurements and simulation at P1 P2 and P3 for Raster strategy (a) and Zigzag strategy (b).

The results demonstrates that the temperature curves obtained from the finite element model correspond well to those measured in the experiment. The average error between the simulated and the experimental values is about 5\%. However, the gap is higher in the first peaks. Possible sources of such error could be the thermal inertia of the thermocouples.

\section{Conclusions}

In this study, a new metal deposition technique is proposed in finite element modeling of WAAM process. This element deposition technique enables, for each deposition time-step, to add new elements to the previous mesh, following the passage of the heat source. Thus, allowing to model both material and heat input, and to better consider heat exchange in the external surfaces. The proposed modeling technique is validated through an experimental test case, where simulated results are shown to be in agreement with the experimental curves. In the future, the proposed simulation is to be used to better understand and predict the thermal related defects in parts fabricated in WAAM.

\section{References}

1. Beraud, N.: Fabrication Assistée par Ordinateur pour le procédé EBM. Thèse, Université Grenoble Alpes (2016) 
2. Vayre, B., Vignat, F., Villeneuve, F.: Metallic additive manufacturing: state-of-the-art review and prospects. Mech. Ind. 13(2), 89-96 (2012)

3. Goldak, J., Chakravarti, A., Bibby, M.: A new element model for welding heat source. Metall. Trans. B. 15B, 299-305 (1984)

4. Xiong, J., Lei, Y., Li, R.: Finite element analysis and experimental validation of thermal behavior for thin-walled parts in GMAW-based additive manufacturing with various substrate preheating temperatures. Appl. Therm. Eng. 126, 43-52 (2017)

5. Xiong, J., Li, R., Lei, Y., Chen, H.: Heat propagation of circular thin-walled parts fabricated in additive manufacturing using gas metal arc welding. J. Mater. Process. Technol. 251, 12-19 (2018)

6. Montevecchi, F., Venturini, G., Scippa, A., Campatelli, G.: Finite element modelling of wirearc-additive-manufacturing process. Procedia CIRP 55, 109-114 (2016)

7. Montevecchi, F., Venturini, G., Grossi, N., Scippa, A., Campatelli, G.: Finite element mesh coarsening for effective distortion prediction in wire arc additive manufacturing. Addit. Manuf. 18, 145-155 (2017)

8. Michaleris, P.: Modeling metal deposition in heat transfer analyses of additive manufacturing processes. Finite Elem. Anal. Des. 86, 51-60 (2014)

9. Ding, J., et al.: Thermo-mechanical analysis of wire and arc additive layer manufacturing process on large multi-layer parts. Comput. Mater. Sci. 50, 3315-3322 (2011)

10. Hu, J., Tsai, H.L.: Heat and mass transfer in gas metal arc welding. Part I: the arc. Int. J. Heat Mass Trans. 50(5-6), 833-846 (2007)

Open Access This chapter is licensed under the terms of the Creative Commons Attribution 4.0 International License (http://creativecommons.org/licenses/by/4.0/), which permits use, sharing, adaptation, distribution and reproduction in any medium or format, as long as you give appropriate credit to the original author(s) and the source, provide a link to the Creative Commons license and indicate if changes were made.

The images or other third party material in this chapter are included in the chapter's Creative Commons license, unless indicated otherwise in a credit line to the material. If material is not included in the chapter's Creative Commons license and your intended use is not permitted by statutory regulation or exceeds the permitted use, you will need to obtain permission directly from the copyright holder.

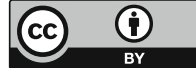

